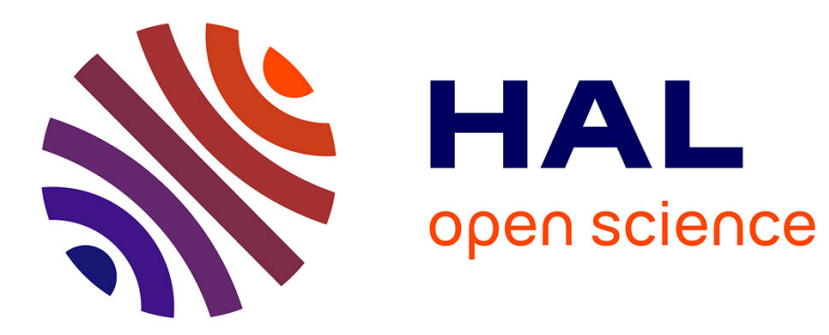

\title{
La détresse respiratoire
}

Michèle Benhaim

\section{To cite this version:}

Michèle Benhaim. La détresse respiratoire. Figures de la psychanalyse, 2008, 10.3917/fp.016.0163 . hal-01429364

\section{HAL Id: hal-01429364 https://hal-amu.archives-ouvertes.fr/hal-01429364}

Submitted on 7 Jan 2017

HAL is a multi-disciplinary open access archive for the deposit and dissemination of scientific research documents, whether they are published or not. The documents may come from teaching and research institutions in France or abroad, or from public or private research centers.
L'archive ouverte pluridisciplinaire HAL, est destinée au dépôt et à la diffusion de documents scientifiques de niveau recherche, publiés ou non, émanant des établissements d'enseignement et de recherche français ou étrangers, des laboratoires publics ou privés. 


\title{
LA DÉTRESSE RESPIRATOIRE
}

Michèle Benhaïm

\author{
ERES | «Figures de la psychanalyse »
}

2007/2 n 16 | pages 163 à 171

ISSN 1623-3883

ISBN 9782749208978

Article disponible en ligne à l'adresse :

http://www.cairn.info/revue-figures-de-la-psy-2007-2-page-163.htm

\section{Pour citer cet article :}

Michèle Benhaïm, «La détresse respiratoire 》, Figures de la psychanalyse 2007/2 (n¹6), p. 163-171.

DOI 10.3917/fp.016.0163

Distribution électronique Cairn.info pour ERES.

(c) ERES. Tous droits réservés pour tous pays.

La reproduction ou représentation de cet article, notamment par photocopie, n'est autorisée que dans les limites des conditions générales d'utilisation du site ou, le cas échéant, des conditions générales de la licence souscrite par votre établissement. Toute autre reproduction ou représentation, en tout ou partie, sous quelque forme et de quelque manière que ce soit, est interdite sauf accord préalable et écrit de l'éditeur, en dehors des cas prévus par la législation en vigueur en France. Il est précisé que son stockage dans une base de données est également interdit. 


\section{La détresse respiratoire}

\section{- Michèle Benhaïm •}

Difficile de nier que la détresse est l'élément de Kafka, c'est son espace, c'est son temps. Kafka écrit du lieu même de la détresse, d'un monde froid et tourmenté. Est-ce une détresse sans espoir ? Chez Kafka, même l'espoir semble participer de la détresse car cela l'empêche de se rassasier du désespoir. " Prends-moi, moi qui ne suis qu'un entrelacement de folie et de douleur ", parole de détresse adressée à Felice Bauer de Marienbad en 1916. Kafka est inconsolable... en proie à une " inimaginable tristesse ", à une " détresse déchirante ".

Pour évoquer cette détresse, nous pouvons interroger et articuler quelques éléments de I'histoire de Kafka avec quelques-uns de ses textes, notamment Le verdict, la Lettre au père et La métamorphose, textes où espoir et détresse se répondent sans fin au sein d'un désastre absolu. $Y$ sont à l'œuvre des questions relatives à la mère du côté d'un deuil impossible, en suspens, un appel au Nom-duPère, comme dans Le verdict puis dans la Lettre au père, où ce dernier apparaît comme un fantoche, un imposteur, lui-même, on le verra, soumis à la loi maternelle, au corps enfin, dont on imagine la douleur dans laquelle il s'est perdu à la fin de sa vie, chez Kafka, cette détresse corporelle dont on sait le retentissement et surtout l'origine chez le nourrisson engagé dans une position originaire de radicale impuissance, dans la nécessité de l'appel à l'autre pour survivre. Si l'autre n'est pas là, pour Kafka, il ne peut être que détruit. Là, surgit la détresse, ouverture sans fond, espace psychique bien au-delà de l'angoisse, qui laissera Kafka sans voix à la veille de sa mort, dans une ouverture désespérée sur l'autre.

Si Kafka ne cesse d'écrire, jusqu'au désespoir, que vivre est une aventure impossible, que vivre c'est être condamné à vivre, c'est que sa détresse est encryptée. Un des éléments de compréhension de cet abîme réside peut-être dans les enfants morts de Julie, la mère de Kafka, encastrés pour toujours dans sa psyché et dans son corps.

Reprenons quelques éléments d'histoire familiale. Quand Julie, la mère de Franz, a 3 ans, sa mère Esther meurt du typhus. À partir de ce jour, la mère 
d'Esther, Sarah, devient mélancolique, cesse de prendre toute nourriture et ne parle plus à personne. La mort interrompt toute parole. "Ça n'a pas de nom ", dira Kafka. Un jour Sarah disparaît et l'on retrouve son corps noyé dans l'Elbe, à jamais muet. Seul le corps a parlé dans une langue close qui se suffit à elle-même. Pour elle, le temps s'est arrêté à la mort de sa fille. Julie a alors 4 ans. Voici deux morts tues, sans mots, sans nom, sans deuil, dans un temps suspendu.

Dans son Journal, Kafka dit : «II faudrait que je puisse inventer des mots propres à chasser l'odeur de cadavre. "Plus loin : "Le fleuve se soulève de dégoût, se met à couler à rebours et rejette les morts dans la vie. " Entre 1883, naissance de Franz, et 1889, naissance de sa sœur Elli, deux nouvelles morts : les deux frères Georg et Heinrich. Franz a 2 ans quand naît Georg qui mourra à l'âge de 6 mois d'une complication de la rougeole, puis 4 ans quand Heinrich connaîtra le même destin à l'âge de 18 mois, des suites d'une otite. La mort des frères rend la mère dépressive et la maison mélancolique. Répétition dans le réel et ce, dans un temps où la parole pour Franz est en voie d'établissement et où le travail de représentabilité psychique ne peut vraisemblablement encore s'accomplir. Les femmes meurent, puis les garçons. Silence sur les morts. Silence dont on sait comme il peut marquer la langue maternelle d'un trou qui sera transmis à l'enfant, peut-être en plein corps.

Comme le soulignent G. Lévy et S. Sabinus dans Le corps dans la tête, Franz est tombe de la mère, son silence, le lieu de sépulture de ses morts. Son corps sera le lieu d'une ultime métamorphose : sa liquéfaction hémoptysique. "Je suis comme ma propre pierre tombale. "

Julie cautionne le déni de la mère. Trouve-t-elle en son fils le corps où déposer cet héritage ? Le trou dans le poumon comme reflet du trou dans la mémoire, la détresse de Kafka s'épuise dans un deuil impossible jusqu'à se taire à son tour (il chuchote à la fin de sa vie comme en témoignent ses Lettres à ses parents) et ne plus pouvoir respirer. II écrit à Milena : " Je suis spirituellement malade, la maladie des poumons c'est seulement la maladie spirituelle qui est sortie de ses rives. " La « blessure " de ses poumons n'est qu'un " symbole ", d'où sa défiance à l'égard des médecins.

L'identification, Freud et Lacan nous l'ont enseigné, c'est l'inscription d'un trait sur un corps. Ici son ratage semble s'originer dans le deuil, son effet s'inscrire dans le corps malade.

À Milena il écrit : " Je devrais couper avec mes dents cette langue qui voudrait parler... le seul moyen de vivre est de nous taire. " À l'origine du silence qui rend le deuil impossible, Sarah qui s'est tue pour toujours. 
Julie puis Franz, à peu près au même âge, vont être pris répétitivement par la double mort, pris dans l'impossible d'un deuil qui signifie qu'un temps s'arrête et se fige, nul temps pour pleurer et assumer les pertes. Alors Julie pourrait être pour Franz au centre du drame. Les Lettres à ses parents, écrites les deux années qui précèdent sa mort, ne s'adressent en réalité qu'à elle. "Tu n'as pas à te faire de souci à propos de la concurrence, en matière de sollicitude, chère maman, tu conserveras ta place ", lui écrit-il, évoquant Dora Dymant. À sa mère, souterrainement, s'adresse sa détresse. Lointaine, maladroite à manifester sa tendresse, sa mère l'aime à sa manière, d'un amour " exactement aussi grand que son incompréhension ".

Franz est le seul garçon survivant. II est fait silence sur ce secret.

Condamné en sursis, destiné à rejoindre les disparus. Mort-vivant. Pour Julie tout semble confus, les places, les limites, les morts, les vivants. Le flou des limites entre les morts et les vivants peut aboutir à une contamination réciproque : les morts refusent de mourir et reviennent, les vivants sont un peu morts... Julie semble résoudre la confusion en choisissant son enfant vivant pour y garder ses enfants morts. L'enfant vivant ne nourrit-il pas les enfants morts et ne leur redonne-t-il pas de la sorte la vie, ce que la mère s'est montrée incapable de faire ? Est-ce le fantasme qui traverserait ce choix ? Doublé du bénéfice de restaurer du même coup cette mère qui laisse mourir ses enfants ? "Chienne puante, mère prolifique, déjà putréfiée par endroits mais qui signifiait tout pour moi dans mon enfance. "

Tandis que les morts ne se laissent pas tuer, Kafka ne peut souffler, condamné à enterrer sans cesse en lui ce qui ne cesse de le hanter. "Nous ne mourrons pas, voilà la vérité, mais il en résulte que nous ne vivons pas non plus, nous sommes morts de notre vivant, nous sommes essentiellement des survivants. " Comment échapper au retour inéluctable des frères morts ? "Cette faute, j'ai dû la commettre, sinon... " La faute d'avoir survécu ? Une faute qu'il n'a pas commise qui le condamne à vivre aussi bien qu'à mourir. " Je suis comme un enfant qui a fait quelque chose de très mal et qui se trouve maintenant devant sa mère, et pleure, et pleure. " Kafka pleure deux fois, pour les deux frères...

Dans Le Verdict, on note que la parole du père " ta mère " est devenue " notre chère maman " ou encore " tu as souillé le souvenir de notre mère ". La Mère est toute-puissante, sur elle repose la force paternelle, «ta mère $\mathrm{m}$ 'a passé sa force ". Alors que chute la loi paternelle, le héros est condamné, comme Sarah, à la noyade. Le fils est condamné et le père est effondré. " Je te condamne à 
présent à la mort par noyade. Georg se sentit projeté hors de la chambre, d'où il emporta encore dans ses oreilles le fracas que faisait derrière lui son père en s'effondrant sur le lit. " La noyade, destin de Sarah, la mère, est un lieu signifiant dans la parole maternelle.

Franz Kafka se définit lui-même comme sujet hors temps et hors discours. II écrit à Felice : "Je n'ai aucune mémoire, je ne peux pas penser, je ne sais pas parler. " "Est-il possible de penser quelque chose d'inconsolable ? À partir d'un certain point, il n'y a plus de retour, c'est ce point qu'il faut atteindre. " C'est de la mère que l'on apprend la parole. C'est elle qui pourvoit l'enfant en signifiants lui permettant de la sorte de symboliser la Chose.

Le Verdict annonce l'effet de l'impossible perte. Le récit s'inaugure d'une annonce de fiançailles qui ne trouve pas d'adresse. Pour se fiancer, il faut manquer de mère, pour se marier, il faut renoncer à la mère. Le destinataire tout comme la parole paternelle se dérobe. Tombe le verdict : " On ne se marie pas. » Alors la lettre demeure encore sans destinataire, morte, le héros en effet court se noyer, sa lettre en poche. Difficile de ne pas associer ici à l'anecdote que Franz raconte à Felice : sa mère un jour s'empare à son insu d'une lettre de Felice. Après ce vol, elle lit la lettre et écrit à Félice.

On a le sentiment que dans ses récits comme dans sa vie, Kafka anticipe que seul le silence répondra à l'appel. Appel au père qui est à la fois appel à sa reconnaissance et appel à sa médiation.

Dans son Journal, Kafka écrit: "J'envoie ma lettre à un mort qui ne la lira pas. " À Felice : "Si tu lui parles de moi, fais-le comme si j'étais mort. "

Le Verdict contient cet appel au Nom-du-Père, à une loi de filiation qui permettrait, dans le mariage, la transmission du nom.

La Lettre au père nous confronte à la rencontre entre un fils et un père comme rencontre toujours manquée. Kafka y construit et y déconstruit sans cesse le père comme pour tenter de répondre à l'énigme de sa fonction. Souvenonsnous que le prétexte de cette longue lettre est l'annulation de son projet de mariage avec Julie Wohryzek. Son père considérait cette union comme médiocre. Franz abandonne son projet de mariage et pense au suicide. De la rue où il loge à Prague, qui ouvre sur le fleuve, il dit qu'elle est « une rue tremplin pour les candidats au suicide ". Sarah... Georg du Verdict...

La Lettre au Père n'arrivera jamais à son destinataire. Elle sera déposée chez Milena en 1920. Elle constitue le modèle même d'un appel sans réponse possible. 
Parce que c'est une folie de croire en une reconnaissance possible, en l'existence d'un destinataire, d'un dépositaire capable de recueillir les mots de la détresse. Une autre lettre à un autre père, celui de Felice, que Franz écrit en 1913, n'arrivera jamais à son destinataire. Encore une lettre volée, muette que Kafka envoie à Felice et qu'elle ne remet pas à son père. L'appel échoue encore.

Confronté à cet impossible de sa rencontre avec l'Autre sexe, Kafka en appelle au père et c'est la répétition du symptôme qui questionne la transmission de la loi symbolique. Franz Kafka décrit dans la Lettre au Père le mariage comme « le plus grand effroi de ma vie... l'épreuve la plus inexorable de toutes ».

Si dans Le Verdict, le père méprisait la fiancée " c'est parce qu'elle a levé ses jupes, cette dinde répugnante, que tu as profané la mémoire de notre mère ", dans la Lettre au père, on apprend que de Julie Worhyzek, le père disait: " Je suppose qu'elle a mis quelque corsage choisi avec recherche comme les juives de Prague s'entendent à le faire, et là-dessus, tu l'as épousée. "

Jusqu'au bout de la Lettre au père, Kafka tente l'appel, faisant de cette lettre une lettre d'amour. "Le sentiment exclusif ressenti par l'enfant est remplacé en partie par une certaine connaissance de notre détresse à tous les deux ", lui écritil. Ultime tentative de rompre le silence, mais tout est faux, "tu faisais semblant ", tout est tromperie. Celui auquel elle s'adresse n'existe pas. Comme l'ami du verdict, le père est faux. Comme dans Le procès, les signifiants qui représentent la loi sont absurdes. Le silence maternel recouvre le vide d'un père. La révélation du verdict c'est que l'ami n'a jamais existé, ni aucun autre destinataire. II n'y a pas plus de père que d'ami. Rien ne peut venir rompre le nœud inextricable entre la mère et l'enfant. " Je voudrais que tu ne fusses pas au monde et que je loge entièrement en toi, l'un de nous est de trop ici. La séparation en deux êtres distincts est intolérable ", écrit-il à Felice en 1913... II dit ailleurs de la tuberculose " nous ne pouvons pas rester en vie tous les deux ". Tant pis, Franz doit écrire ces lettres, c'est tout ce qu'il sait : "Il est bien possible que j'envoie ma lettre à un mort qui ne la lira pas... or moi, il faut que j'écrive ma lettre. »

Dans La métamorphose, la mère ne peut supporter l'existence de son fils que mort ou absent. " J'ai le droit de mourir », pense Grégoire enseveli. Le seul droit qui lui soit reconnu. De ce texte Franz Kafka dira : "La métamorphose, une petite histoire qui m'est venue à l'esprit tandis que j'étais couché en pleine détresse et qui m'obsède au plus profond de moi-même. " Grégoire s'écoute changer de voix, se métamorphoser, piaulement de gorge comme dans la tuberculose. Si la sœur de Grégoire dit: "Je ne peux pas prononcer le nom de mon 
frère en parlant du monstre qu'il y a ici ", Franz nomme sa peur sans nom, " un groupe de noms qui finit par K. ne peut pas respirer ".

Franz, entre deux séries de morts occupe une place impossible, et pourtant, comme Grégoire, nécessaire à l'équilibre de tous les autres.

Toute la famille regroupée autour de la chambre de Grégoire cherche à lui arracher un signe de vie. Mais Grégoire ne peut pas bouger, prisonnier de sa carapace. S'il parle, il sauve tout le monde, il échappe à la métamorphose, il sort de l'insensé, du silence. Mais quand Grégoire essaie de trouver un destinataire qui pourrait soutenir sa parole, il se confronte à la famille qui s'affaire. Seule la mère pourrait le délivrer du secret enfoui en lui mais, au seuil de voir Grégoire, elle s'évanouit, son visage est " introuvable ", visage de la mère qui, à travers sa fonction de signal, ouvre à la fonction symbolique. Le voir, c'est comme l'entendre, le deuil impossible demeure insoutenable.

Il y a un renversement dans le texte : au début la porte de la chambre de Grégoire est fermée de l'intérieur. C'est lui le gardien de la famille. Après la rencontre, ça bascule. Il est enfermé comme le secret de famille dans une chambre devenue tombe. Peu à peu, le voici devenu trou de silence quand la famille l'évoque, signifiant exclu, innommable, irreprésentable pour la mère, " ça » pour la sœur.

S'être tu ne suffit pas, il faut qu'il soit enterré avec les morts. Là réside l'impossible tâche du travail de deuil, enterrer le mort. "Mais lorsque je n'écrivais pas, j'étais par terre, tout juste bon à être balayé ", écrit-il à Felice. Comme Grégoire ?... « Devant les enfants, je préfère fermer les yeux », écrit Kafka. Ne plus voir, ne plus savoir, oublier, silence familial devant la porte close de Grégoire. Seule rencontre envisageable, celle avec le signifiant de la mort. Dans La métamorphose, pas de deuil, pas de mort, juste une détresse respiratoire, une gêne au poumon, chez la mère. La sœur se charge de tout, y compris du deuil de la mère. Quand prise d'une crise de larmes, ses pleurs coulent sur le visage de la mère, elle les essuie machinalement de la main. L'enfant et la mère se donnent l'un l'autre un visage pour pleurer.

Quelques mois après la certitude du verdict de la tuberculose, Franz rompt définitivement ses fiançailles avec Felice. II arrête au même moment d'écrire pendant trois ans. La maladie relaie la relation amoureuse et les mots muets s'y tracent. La lésion au poumon semble prendre le statut de l'inscrit. C'est pendant ces trois années qu'a lieu le mariage manqué avec Julie Worhyzek et l'écriture de la Lettre au père. Kafka écrit : " Je suis aujourd'hui, avec la tuberculose, dans le 
même rapport qu'un enfant avec les jupes de sa mère auxquelles il s'accroche. Si ma maladie me vient de ma mère, l'image est encore plus juste, et ma mère, très au-dessous de sa compréhension de la chose, $\mathrm{m}$ 'aurait encore rendu ce service dans sa sollicitude infinie. "

Milena, "Maman Milena », comme il appellera sa fiancée. essaiera de faire des projets, d'apaiser la détresse de Kafka. Elle se heurte à l'intransigeance de son désespoir, à la force de son silence et de sa solitude. Kafka ne voit que ça à aimer. De son angoisse il dit : "Puisqu'elle est ma meilleure part, elle est aussi peut-être la seule chose que tu aimes qui fut digne d'amour. " Tout autre est disqualifié dans sa tentative de répondre au désespoir, d'en devenir l'objet.

Finalement, détresse, angoisse, inquiétude, tourment forment le destin dont Kafka a plus que conscience et qui, justement, à partir de cette conscience de luimême comme un être abîmé, soufflé par le néant, n'en demeure pas moins ce qui le rend digne d'être aimé. L'angoisse est là, tout le temps. Le désir est angoisse. La détresse est infinie. II écrit à Milena, " moi, l'animal de la forêt... je gisais quelque part dans une fosse boueuse (boueuse seulement par suite de ma présence) ".

Toute rencontre chez Kafka se paie de détresse. Quand il écrit Le terrier, le soir, avant de s'installer à son bureau, il est mélancolique comme toujours les derniers temps lorsqu'il se préparait à l'écriture. C'est dans l'insomnie, la nuit sans sommeil, cette situation impossible, ce drame de chacune de ses nuits, que Kafka écrit. Écrire comme une recherche de continuité absolue, peut-être une recherche de continuité d'être. "Écrire est mon unique possibilité d'existence. " Il est comme à la fois impossible de vivre et impossible d'en finir.

Kafka est condamné à la " malédiction » d'écrire. " Je ne puis parler de l'essentiel, il est même pour moi enfermé dans l'obscurité de ma poitrine : c'est là qu'il se tient à côté de la maladie, sur le même lit commun. " "Ce n'est plus la tuberculose qu'on étend sur une chaise longue et qu'on soigne, mais une arme, dont la nécessité extérieure subsistera aussi longtemps que je resterai en vie. Et les deux ne peuvent ensemble rester en vie. "Organes de respiration atteints, Franz ne peut plus ni boire, ni manger, ni parler. II n'y aura ni Amérique, ni Palestine, mais seulement les fantômes, la maladie, la mort. "Je rôde quelque part, sur le parvis de l'enfer. "

Pour conclure sur cette hypothèse autour du deuil impossible, ces morts évoquées seraient comme inaperçues et on peut se demander avec Pierre Fédida quel déni peut bien survenir qui rend la mort inaperçue. Ne pas apercevoir la 
mort, c'est laisser se recouvrir les affects douloureux avant qu'ils n'apparaissent. Rien n'a vraisemblablement échappé à Franz de la disparition et de la mort. Jean Genet disait que " l'artiste dispose du pouvoir étrange de pénétrer le domaine de la mort, de recueillir le souffle indistinct des ombres que sont ces morts devenus ".

Écrivain jusqu'au dernier instant, Kafka s'est laissé emmurer par ceux auxquels il a été refusé sépultures. Sa détresse s'achève dans la constitution d'un tombeau pour l'autre dans le corps. Kafka écrit d'en deçà de la mort, comme s'il revenait de la mort, comme s'il l'avait déjà traversée. II n'y va pas. II en revient. À propos des passages de son œuvre qu'il considère comme les plus réussis, il écrit : "Il s'agit toujours de quelqu'un qui meurt. " II rêve sa mort comme d'autres rêvent leur vie. "N'avoir jamais que le désir de mourir et s'accrocher encore, cela seul est l'amour. »

Pourtant, évidemment, Kafka n'a pas connu la Shoah. Pourtant toutes les lettres qu'il a écrites à Dora ont été confisquées puis détruites par la Gestapo. Pourtant Milena est morte à Treblinka. Ainsi que ses trois sœurs, Elli, Valli et Ottla.

Franz n'a pas connu la Shoah et l'anéantissement. Le monde de Kafka est sombre et glacé, ses nuits sont sans sommeil, sa tristesse est sans fond, à mourir. La Colonie pénitentiaire, la " vermine » et l'anéantissement dans La métamorphose sont devenus réalités peu après sa mort, souligne George Steiner. À Auschwitz une valise porte l'inscription "Marie Kafka ». Je n'ai pas retrouvé de Marie dans sa famille. Kafka est seul, si seul. Dora ne peut s'arracher à son corps, comme je n'ai pu me décider à détacher mon regard de cette pauvre valise. Elle veut le protéger, encore, des fantômes. J'ai eu du mal à retrouver mon souffle. "Mais moi, je m'en vais ", souffle Franz Kafka.

"Après la mort d'un être humain, écrivait-il en 1918 dans son journal, un silence spécial et bienfaisant se fait pour un temps, même sur terre, en ce qui concerne la personne du mort, une fièvre terrestre a cessé, on ne voit plus se poursuivre une agonie, une erreur paraît supprimée, même pour les vivants, c'est une occasion de reprendre souffle, ce qui explique aussi qu'on ouvre les fenêtres des chambres mortuaires, jusqu'au moment où tout cela se révèle n'être qu'une apparence et où commencent lamentations et douleurs. " 


\section{RÉSUMÉ}

La détresse est l'élément de Kafka ; elle sera analysée à partir de l'œuvre de Kafka, notamment des textes Le verdict, la Lettre au père et La métamorphose, textes où espoir et détresse se répondent sans fin au sein d'un désastre absolu. Les questions sont relatives à la mère du côté d'un deuil impossible, en suspens, un appel au Nom-du-Père et au corps dont on imagine la douleur dans laquelle il s'est perdu à la fin de sa vie.

Un des éléments de compréhension de cet abîme réside peut-être dans les enfants morts de Julie, la mère de Kafka, encastrés pour toujours dans sa psyché et dans son corps et témoignant d'un deuil impossible.

\section{MotS-CLÉS}

Détresse respiratoire, deuil impossible, mère, douleur, corps.

\section{SUMmaRY}

The distress is the element of Kafka and will be analyzed from Kafka's work, including texts The Verdict, the Letter to the father and The Metamorphosis in which hope and distress endless respond each other in an absolute disaster. The questions are related to the mother's impossible mourning, to the name of the Father and to the body of which it is easy to imagine the pain in which Kafka lost at the end of his life. One element of understanding of this abyss may lie in Julie's dead children, the mother of Kafka, recessed forever in his psyche and in his body and showing an impossible mourning.

\section{KeY-WORDS}

Respiratory distress, impossible mourning, mother, pain, body. 